\title{
Detecting Moving Targets in Dual-Channel High Resolution Spaceborne SAR Images With a Compound Detection Scheme
}

\author{
Diana Weihing ${ }^{1}$, Stefan Hinz ${ }^{1}$ \\ ${ }^{1}$ Remote Sensing Technology \\ Technische Universität München \\ Arcisstr. 21, 80333 München, Germany \\ Email: Diana.Weihing@bv.tum.de
}

\author{
Franz Meyer ${ }^{2}$, Steffen Suchandt ${ }^{2}$, Richard Bamler ${ }^{1,2}$ \\ ${ }^{2}$ Remote Sensing Technology Institute (IMF) \\ German Aerospace Center (DLR) \\ Oberpfafffenhofen, 82234 Wessling, Germany
}

\begin{abstract}
Traffic data acquisition from space has evolved to an important task over the last years. Future SAR satellite missions will provide high resolution dual-channel SAR data and therefore a possibilty to collect traffic parameters of a large area from space. In this paper a detection approach for vehicles will be presented, which considers simultaneously the effects moving objects suffer from in the SAR image. The performance of the proposed detection scheme is analyzed using experimental airborne SAR data.
\end{abstract}

\section{INTRODUCTION}

\section{A. Motivation}

Due to increasing traffic, traffic control and traffic data acquisition have become an important task. To derive statistical parameters of the situation on the road network, an observation of large areas is necessary. Upcoming satellite SAR missions (like TerraSAR-X and RADARSAT-2) entail potential for such applications. In addition to the large coverage of a spaceborne SAR sensor, its independence regarding daytime and weather conditions make SAR an interesting tool for traffic data acquisition. These sensors will provide SAR images up to 1 $\mathrm{m}$ resolution and, in the Dual Receive Antenna mode, two SAR images of the same scene within a small timeframe. This enables interferometric measurements for vehicle detection.

\section{B. Related work}

Detection and velocity estimation of vehicles in spaceborne SAR data are a challenging task. Since moving objects suffer from special effects in the SAR processing algorithm, specific methods for detecting vehicles are required. In military research this problem is known as Ground Moving Object Indication (GMTI). If more than two channels are available the use of Space Time Adaptive Processing (STAP) is the optimal method [1], [2]. In the case of two-channel systems, like TerraSAR-X or the Canadian RADARSAT-2, interferometric approaches can be used for detecting vehicles. Along-Track Interferometry (ATI) [3] and the so-called Displaced Phase Center Array (DPCA) method are the classical methods to do so. They can be regarded as an approximation of the limiting case of STAP. In the ATI technique an interferogram is formed from the two SAR images by complex conjugate multiplication, whereas in the DPCA processing the two calibrated SAR images are subtracted from each other. The interferometric phase in ATI and the magnitude of the result in DPCA are evaluated for detection [4]. These detections are done based on a constant false alarm rate scheme. In [5] these approaches have been extended by integrating apriori information, GIS data of road networks. However, e.g. ATI can only be applied if the motion of the vehicle affects the interferometric phase, which is not the case if vehicles are moving in along-track direction. To estimate ground moving parameters for vehicles travelling in along-track, one method is to apply filterbanks with differently designed matched filters [6], [7].

The presented detection approach in this paper considers simultaneously the effects in SAR images, which are caused by the vehicle's motion in across- and along-track. The scheme is derived from statistical detection theory and its principle relies on comparing an expected signal with the actual measurement in the SAR data. Therefore, different information are combined in this detection algorithm. The expected signal, the measured signal and their variances are included to decide wheter a vehicle is present or not.

In the next section a short summary is given of the different effects in SAR images caused by the vehicle's motion. The proposed likelihood ratio detector is explained in Sect. III. Afterwards the performance of this detector is analysed using experimental airborne SAR data (E-SAR from DLR) in Sect. IV and conclusions are drawn in Sect. V.

\section{SAR IMAGING OF MOVING OBJECTS}

In an air- or spaceborne SAR imaging process a radar scans the earth in a side-looking fashion during its flight over the scene. The radar is flying in a certain altitude $h$ along the $\mathrm{x}$-axis, also referred to azimuth direction or along-track. The $y$-axis, which is oriented perpendicular to the flight path, is usually refered to as ground range or across-track direction. While the sensor is moving, it transmits microwave pulses 
at constant intervals given by the Pulse Repetition Frequency (PRF) and receives the echoes backscattered on the ground. The received echoes are assembled in a raw data matrix lineby-line. Every scatterer contributes to the data with a certain signal. The phase history of one scatterer, also called azimuth chirp, can be written as:

$$
h(t)=\exp \left(-j \frac{4 \pi}{\lambda} R(t)\right)=\exp \left(j \pi F M t^{2}\right)
$$

where $F M=-\frac{2}{\lambda} \frac{v_{s a t} v_{b}}{R_{0}}$ is the frequency modulation (FM) rate of the azimuth chirp.

To form the final SAR image of the scene, a matched filter algorithm is applied to the raw data matrix. The filter for azimuth focusing is defined by:

$$
s(t)=\exp \left(-j \pi F M t^{2}\right)
$$

If a scatterer is moving during the data acquisition, the phase history $h(t)$ of this point will change and the scatterer will not be imaged "correctly" after the focusing algorithm $h(t) * s(t)$.

\section{A. Motion in across-track}

If the scatterer is moving in across-track, it causes a linear trend of the phase history. When convolving its phase history with the matched filter, it results in a shift of the object in the image. The shift in time-domain can be expressed in seconds or in meters:

$$
t_{\text {shift }}=\frac{2 v_{\text {los }}}{\lambda F M}[s] ; \quad \Delta_{\text {azimuth }}=-R \frac{v_{\text {los }}}{v_{\text {sat }}}[m]
$$

Hence, the vehicle is not imaged at its correct position, but displaced in azimuth direction depending on its line-of-sight velocity $v_{l o s}$.

Across-track motion not only causes a shift of the position of a vehicle in the image, but also an interferometric phase if the data is acquired in an along-track interferometric mode. The interferometric phase $\phi$ is defined as the phase difference of the two images, which are acquired within a small time frame, and is given by:

$$
\phi=\left(\frac{4 \pi B_{A T I} v_{\text {los }}}{\lambda v_{\text {sat }}}\right) \bmod 2 \pi
$$

which is zero for stationary objects or objects moving only in along-track.

\section{B. Motion in along-track}

In the case the vehicle is moving in along-track, the relative velocity between sensor and scatterer gets changed and hence also the FM rate. Focusing with the matched filter leads to a spread of the signal in the image. The blurring can be approximated by:

$$
\Delta t \approx \frac{P R F}{\delta F M}=2 T_{A} \frac{v_{x 0}}{v_{B}}
$$

This equation shows that a target gets smeared twice the way it moved during the time of illumination $T_{A}$.

\section{Acceleration in across-track}

Like the component of along-track motion, acceleration in across-track mainly causes a spreading of the energy and the target will be smeared in azimuth. The crosstalk of alongtrack motion and across-track acceleration hampers the interpretability of along-track smearing without having additional information available [8], [5].

\section{Acceleration in along-track}

Acceleration in along-track causes an assymetry of the focused point spread function. However, for TerrSAR-X these effects are neglible [9].

\section{Proposed Likelihood Ratio Detector}

As shown in the preceding section a moving object causes an interferometric phase, hence also a displacement, and it causes a blurring of its signal in the SAR image. However, with the knowledge of roads and their directions, it is possible to forecast a signal one expects for certain situations. To decide whether a vehicle is present or not, these expected signals are compared with the actual value in the SAR image.

The mathematical framework for this proposed approach is derived from statistical detection theory. Two hypotheses $H_{0}$ and $H_{1}$ are defined:

$H_{0}$ : only clutter and noise are existent

$H_{1}$ : signal plus clutter and noise are existent

Together with the corresponding probability density functions $f\left(\vec{x} \mid H_{0}\right)$ and $f\left(\vec{x} \mid H_{1}\right)$, these hypotheses allow setting up a likelihood ratio test given by:

$$
\Lambda=\frac{f\left(\vec{x} \mid H_{1}\right)}{f\left(\vec{x} \mid H_{0}\right)}
$$

where

$$
f\left(\vec{x} \mid H_{0}\right)=\frac{1}{\pi^{2}|C|} \exp \left\{-\vec{X}^{H} C^{-1} \vec{X}\right\}
$$

and

$$
f\left(\vec{x} \mid H_{1}\right)=\frac{1}{\pi^{2}|C|} \exp \left\{-(\vec{X}-\vec{S})^{H} C^{-1}(\vec{X}-\vec{S})\right\}
$$

are circular Gaussian Random Processes with $\vec{S}$ being the expected signal, $\vec{X}$ being the measured signal, $C$ being the covariance matrix, and $(*)^{H}$ being the Hermitian Matrix. See e.g. [10] for their derivation.

Finally, from Eqs. 6-8 we can derive the Bayesian decision rule of log-likelihood test, which computes to:

$$
\left|\vec{S}^{H} C^{-1} \vec{X}\right|>\alpha
$$

which is the mathematical framework for the detection scheme. This means an expected signal hidden in clutter is compared with the actual measurement.

In particular, following pieces of information are combined in this detection approach: the expected signal $\vec{S}$, the measured signal $\vec{X}$, as well as their covariances $C$, which are described in more detail below. 
The measured signal consists of the SAR images from the two apertures:

$$
\vec{X}=\left(\begin{array}{c}
X_{1} \\
X_{2}
\end{array}\right)
$$

with the indices standing for the two channels.

For the expected signal, a priori knowledge is required. With the information of road location and road direction from GIS data, an expected phase for each pixel depending on the pixel's distance to the corresponding road segment can be forecasted. With the expected phase $\phi$ the expected signal can be derived:

$$
\vec{S}=\left(\begin{array}{c}
S_{1} \\
S_{2}
\end{array}\right)=\left(\begin{array}{c}
\exp \left\{j \frac{\phi}{2}\right\} \\
\exp \left\{-j \frac{\phi}{2}\right\}
\end{array}\right)
$$

where also the indices significate the two channels. In case of dense road networks, this step must be made for each road segment individually, since the displacement effect may cause overlapping predictions stemming from different roads.

The covariance matrix is defined as in [10]:

$$
C=E\left\{X X^{H}\right\}=\left(\begin{array}{cc}
\bar{I}_{1} & \gamma \bar{I} \\
\gamma^{*} \bar{I} & \bar{I}_{2}
\end{array}\right) \approx \bar{I}\left(\begin{array}{cc}
1 & \gamma \\
\gamma & 1
\end{array}\right)
$$

with $\bar{I}=\sqrt{\bar{I}_{1} \bar{I}_{2}}=\sqrt{E\left[\left|u_{1}\right|^{2}\right] E\left[\left|u_{2}\right|^{2}\right]}$.

However in real scenarios with roads heading in any direction not only an interferometric phase occurs, but also a blurring of the signal over several resolution cells. Thus, neighbouring pixels are included into the mathematical framework to decide whether the considered pixel is a vehicle or not. The possible blurring effect in these adjacent pixels subject to existing along-track motion or across-track acceleration will be adjusted by variation of filters to obtain a focused object and thus an optimized peak-to-sidelobe-ratio for its signal, see Fig. 1a). Therefore, a stack of resulting images processed with different FM rates is evaluated for detection, see Fig. 1b). Every pixel in this stack has to be compared to the threshold $\alpha$, yielding in a decision for $H_{1}$ for the pixel when using the corresponding FM rate.

$$
\max _{F M}\left|\vec{S}^{H} C^{-1} \vec{X}(F M)\right|>\alpha
$$

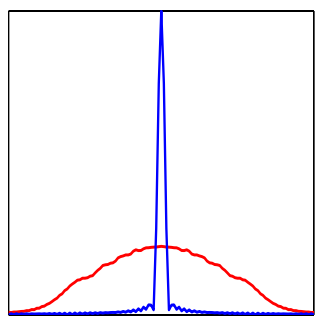

(a)

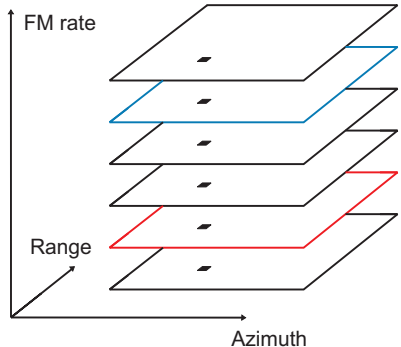

(b)
Fig. 1. a) blurred signal of a vehicle focused with the filter for stationary targets (red curve) and the same signal focused with the corresponding FM rate (blue curve). b) stack of images processed with different FM rates
The threshold that decides whether the evaluated pixel is a vehicle or not, depends on the choice of statistical parameters. The cut-off value for the cumulative density function of the resulting log-likelihood test considering an desired false alarm rate is integrated.

\section{RESULTS}

At the german aerospace center (DLR) a modular traffic processor has already been developed which processes SAR data specifically with the objective of moving vehicle detection [11], [12]. The proposed likelkihood ratio detector has been included additionally to other detection methods into this environment to enable analysis of this detection scheme.

The proposed likelihood ratio detector is tested on real SAR data. DLR's E-SAR data of a flight campaign at Lake Chiemsee, approx. $80 \mathrm{~km}$ east of Munich, has been used. One sees in Fig. 2 a part of the motorway A8 on the southern lakeshore heading nearly in across-track. The detected vehicles at their displaced positions are marked with red rectangles. The triangels are the positions of these vehicles backprojected to the assigned road. These triangles are color-coded regarding their estimated velocity and the range is from blue to red, standing for 0 to $170 \mathrm{~km} / \mathrm{h}$. When projecting these detections back onto the road axis, it is possible to derive parameters for the situation on the road and using them for traffic models.

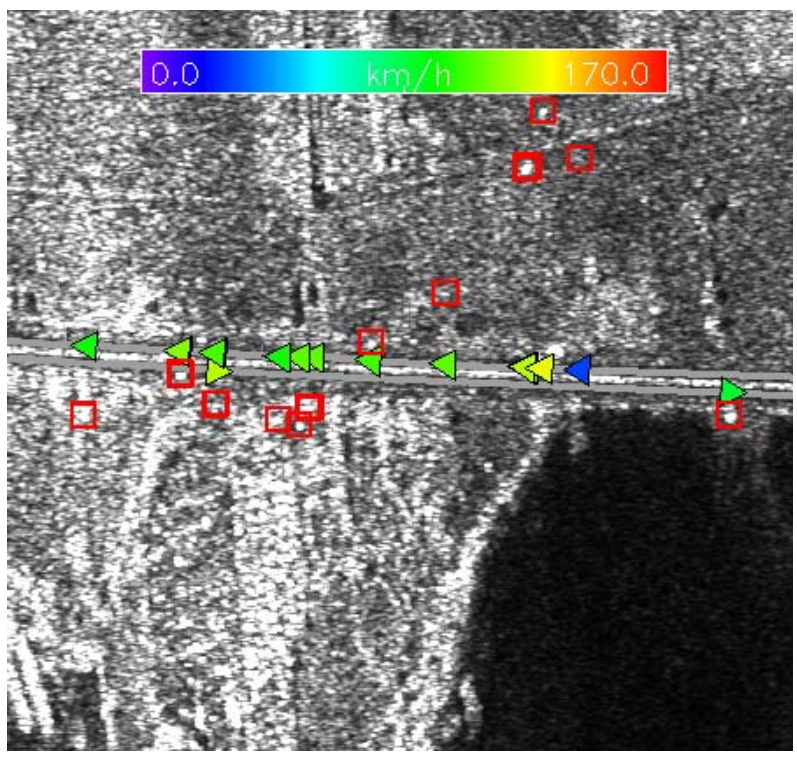

Fig. 2. Detection results using the proposed likelihood ratio detector

During the flight campaign optical images of the same scene have been acquired additionally to enable the verification of the detection results. Therefore, ground truth data of the situation on the road at the time of the SAR data acquisition is available. The positions of the vehicles are measured in the sequence of the optical images. Through "tracking" these vehicles it is possible to estimate their velocities during the SAR data acquisition. The tracks allow to define the velocity of a car at each azimuth time stamp. These reference cars 
are mapped into the SAR image, which is shown in Fig. 3. They are also colorcoded regarding their velocities at the range of 0 to $170 \mathrm{~km} / \mathrm{h}$.

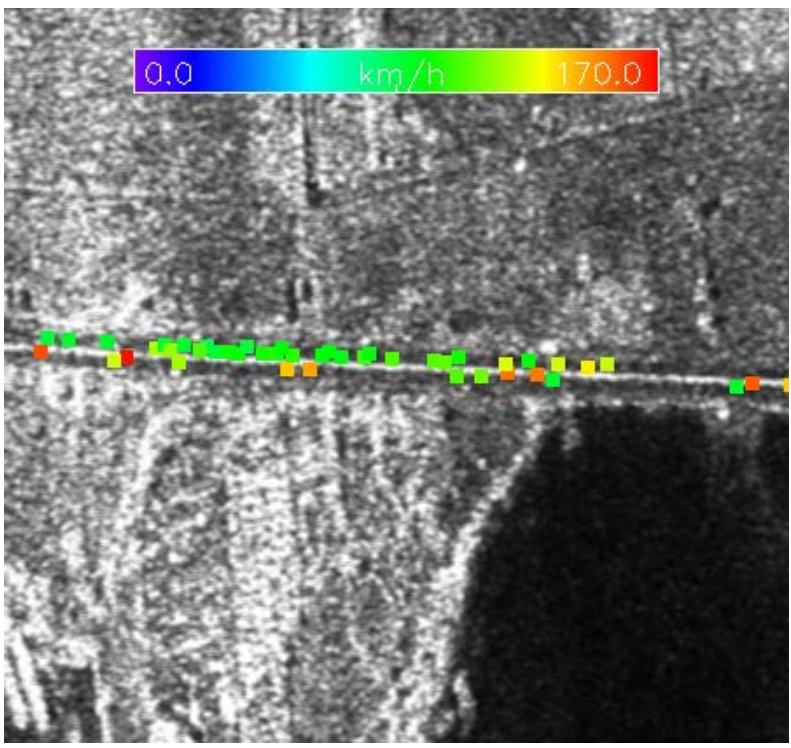

Fig. 3. Reference cars mapped into SAR image, colorcoded reagrding their velocizy

In Tab. I the traffic flow parameters derived from the detections with the likelihood ratio detector are compared to those estimated from the reference data. The vehicles moving on the upper lane from the right hand to the left hand side are considered in this case. On the opposite lane only two vehicles have been detected and hence it is difficult to derive reliable parameters for the situation for this direction of the road. For the first mentioned vehicles in this scene the following parameters, listed in Tab. I, are derived. Comparing the values, e.g. the estimated mean of velocity, a good correspondence can be seen. Hence, even for a lower percent of detections in the SAR data, reliable parameters can be extracted. So, for a part of the motorway additional parameters could be estimated, e.g. the expected duration to pass this road segment.

\begin{tabular}{|l|l|l|l|}
\hline $\begin{array}{l}\text { Traffic flow parame- } \\
\text { ters }\end{array}$ & SAR data & $\begin{array}{l}\text { SAR data } \\
\text { with outlier } \\
\text { rejection }\end{array}$ & optical data \\
\hline mean velocity & $104 \mathrm{~km} / \mathrm{h}$ & $111 \mathrm{~km} / \mathrm{h}$ & $100 \mathrm{~km} / \mathrm{h}$ \\
\hline velocity range & $29-129 \mathrm{~km} / \mathrm{h}$ & $97-129 \mathrm{~km} / \mathrm{h}$ & $81-135 \mathrm{~km} / \mathrm{h}$ \\
\hline number of vehicles & 12 & 11 & 31 \\
\hline detection rate & $39 \%$ & $35 \%$ & $100 \%$ \\
\hline
\end{tabular}

TABLE I

TRAFFIC FLOW PARAMETERS FOR VEHICLES MOVING ON THE UPPER LANE FROM THE RIGHT HAND TO THE LEFT HAND SIDE

Possible false detections, like the vehicle estimated with 29 $\mathrm{km} / \mathrm{h}$, could be eliminated, if considering additional information. Since vehicles in the flow of traffic depend on each other spatially and hence also regarding the velocity, connections between these information can be used to verify outlier.
Therefore, the average velocity and its standard deviation estimated from all detected cars can be used to set up a confidence interval outside of that vehicles are rejected (see third column of Tab. I).

\section{CONCLUSION}

An approach to detect vehicles in SAR data by considering simultaneously the effects due to the vehicle's motion has been proposed in this paper. The preceding results using real airborne SAR data show that it is possible to detect vehicles with the proposed likelihood ratio detector and estimate ground moving parameters for the situation on the road. The derived traffic flow parameters from these results show a good correspondance with those received from optical reference data, even for a low detection rate.

\section{ACKNOWLEDGMENT}

Thanks go to the DLR traffic processor developers for their support and for providing the environment of the traffic processor to enable analyses of the proposed likelihood ratio detector.

\section{REFERENCES}

[1] J. Ender, "Space-time processing for multichannel synthetic aperture radar," Electronics \& Communication Engineering Journal, vol. 11, no. 1, pp. 29-38, 1999.

[2] R. Klemm, Space-time adaptive processing, R. Klemm, Ed. London, United Kingdom: The Institute of Electrical Engineers, 1998.

[3] I. Sikaneta and C. Gierull, "Two-Channel SAR Ground Moving Target Indication for Traffic Monitoring in Urban Terrain," in International Archives of Photogrammetry, Remote Sensing and Spatial Information Sciences, vol. 36, no. 3/W24, 2005, pp. 95-101.

[4] C. Gierull, "Moving Target Detection with Along-Track SAR Interferometry," Defence Research \& Development Canada, Tech. Rep. DRDCOTTAWA-TR-2002-084, 2002.

[5] F. Meyer, S. Hinz, A. Laika, D. Weihing, and R. Bamler, "Performance analysis of the terrasar-x traffic monitoring concept," ISPRS Journal of Photogrammetry and Remote Sensing, vol. 61, no. 3-4, pp. 225-242, 2006.

[6] C. Gierull and I. Sikaneta, "Ground Moving Target Parameter Estimation for Two-Channel SAR," in EUSAR 2004 Conference. Ulm, Germany: on CD, 2004.

[7] D. Weihing, S. Hinz, F. Meyer, A. Laika, and R. Bamler, "Detection of along-track ground moving targets in high resolution spaceborne SAR images," in International Archives of Photogrammetry, Remote Sensing, and Spatial Information Sciences, vol. 36-7, 2006

[8] J. Sharma, C. Gierull, and M. Collins, "Compensating the effects of target acceleration in dual-channel SAR-GMTI," IEEE Processdings Radar, Sonar \& Navigation, vol. 153, pp. 53-62, 2006.

[9] S. Hinz, F. Meyer, M. Eineder, and R. Bamler, "Traffic Monitoring with spaceborne SAR - Theory, simulations, and experiments," in Computer Vision and Image Understanding, to appear, 2007.

[10] R. Bamler and P. Hartl, "Synthetic aperture radar interferometry," Inverse Problems, pp. R1-R54, 1998.

[11] S. Suchandt, M. Eineder, R. Müller, A. Laika, S. Hinz, F. Meyer, and G. Palubinskas, "Development of a GMTI processing system for the extraction of traffic information from TerraSAR-X data," in EUSAR 2006 Conference, on CD, Ed., 2006.

[12] S. Suchandt, M. Eineder, H. Breit, and H. Runge, "Analysis of ground moving objects using SRTM / X-SAR data," ISPRS Journal of Photogrammetry \& Remote Sensing, vol. 61, no. 3-4, pp. 209-224, 2006. 